\title{
Transtornos do sono em pacientes ambulatoriais com depressão
} Sleep disorders in outpatients with depressive disorder

\author{
SARAH LAXHMI ChELlaPpA ${ }^{1}$ \\ John Fontenele Araújo ${ }^{2}$
}

\begin{abstract}
Resumo
Contexto: Os transtornos do sono são uma característica marcante do transtorno depressivo e podem ocorrer nos primeiros estágios da depressão, antecipá-la ou ser um sintoma residual. Objetivo: Avaliar as queixas de transtornos do sono em pacientes ambulatoriais com transtorno depressivo de um hospital geral. Métodos: Foi realizado um estudo observacional, transversal, com uma amostra de 70 pacientes (44 mulheres e 26 homens) com diagnóstico de transtorno depressivo, segundo os critérios da DSM-IV. Os pacientes foram entrevistados e avaliados por meio da ficha de identificação do questionário de hábitos de sono e do Inventário de Depressão de Beck (IDB). Resultados: Neste estudo, 50 (71,3\%) pacientes apresentaram recorrência das queixas de transtornos do sono. A média dos escores obtida no IDB foi 35,83 $\pm 8,85$, com diferenças significativas entre os pacientes com $(38,50 \pm 8,70)$ e sem $(29,60 \pm 7,80)$ recorrência $(\mathrm{p}<0,05)$ e entre pacientes com $1,2,3$ e $>3$ episódios $(\mathrm{p}<0,05)$ de transtornos do sono. Neste estudo, 49 (70\%) pacientes apresentaram insônia e 21 (30\%), sonolência excessiva subjetiva. Houve diferença significativa entre a duração média em meses dos transtornos do sono $(7,16 \pm 2,10)$ e do transtorno depressivo $(6,12 \pm 1,90)(p<0,05)$. Discussão: Na amostra estudada, à recorrência das queixas de transtornos do sono foi elevada e associada significativamente a depressão mais grave. Houve prevalência de insônia, e a duração média dos transtornos do sono foi maior em relação à do transtorno depressivo.
\end{abstract}

Palavras-chave: Transtornos do sono, distúrbio do início e da manutenção do sono, transtornos do sono por sonolência excessiva, transtorno depressivo.

\begin{abstract}
Background: Sleep disorders are a characteristic feature of depressive disorder and can occur in the first stages of depression, anticipate it or act as a residual symptom. Objective: To evaluate sleep disorder complaints in outpatients with depressive disorder from a general hospital. Methods: An observational, cross-sectional study was carried out with a test group of 70 patients (44 women and 26 men) with diagnosis of depressive disorder, according to the DSM-IV criteria. The patients were interviewed and evaluated by the Identification Questionnaire, the Sleep Habits Questionnaire and the Beck Depression Inventory (BDI). Results: In this study, 50 (71.3\%) patients had recurrence of sleep disorder complaints. Mean BDI score was $35.83 \pm 8.85$, with significant differences between patients with $(38.50 \pm 8.70)$ and without $(29.60 \pm 7.80)$ recurrence $(\mathrm{p}<0.05)$ and among patients with $1,2,3$ and $>3$ episodes $(\mathrm{p}<0.05)$. In this study, $49(70 \%)$ patients had insomnia and $21(30 \%)$ had excessive
\end{abstract}

Recebido: 08/01/2006 - Aceito: 13/03/2006

1 Médica e mestranda do Programa de Pós-Graduação em Ciências da Saúde da Universidade Federal do Rio Grande do Norte (UFRN).

2 Médico e professor adjunto do Departamento de Fisiologia e do Programa de Pós-Graduação em Ciências da Saúde da UFRN.

Endereço para correspondência: Sarah Laxhmi Chellappa. Avenida Campos Sales, 414 - 59012-300

- Natal/RN. Fone: (0xx84) 3201-1545. E-mail: sarahlc@ig.com.br 
sleepiness. Significant differences were observed between the mean duration in months of the sleep disorders $(7.16 \pm 2.10)$ and the depressive disorder $(6.12 \pm 1.90)(\mathrm{p}<0.05)$. Conclusion: In the study sample, recurrence of sleep disorder complaints was high and significantly associated with severe depression. Insomnia was

\section{Introdução}

Os transtornos do sono são uma característica marcante do transtorno depressivo, e aproximadamente $80 \%$ dos pacientes com depressão apresentam queixas pertinentes à deterioração tanto da quantidade como da qualidade do sono (Stein e Mellman, 2005). Entre as principais alterações descritas, há uma prevalência da insônia, com acentuada dificuldade de iniciar e/ou manter o sono e despertar precoce pela manhã (Ohayon, 2000; Vaughn e D'Cruz, 2005). As queixas específicas podem incluir despertares noturnos freqüentes, sono não restaurador, redução do sono total e sonhos perturbadores (Bonnet e Arand, 1997; ICSD, 2005). Todavia, em torno de $10 \%$ a $20 \%$ dos pacientes depressivos referem sonolência excessiva, principalmente na depressão grave ou na depressão atípica (Stein e Mellman, 2005).

A associação entre o transtorno depressivo e a insônia é estreita, notadamente quando esta ocorre em quadros depressivos graves (Ford e Cooper-Patrick, 2001), e pode estar associada à marcada ideação suicida (Bernert et al., 2005). Atualmente, a insônia tem sido definida como comórbida em algumas condições médicas, como o transtorno depressivo (NIH-NIMH, 2005).

As principais alterações da arquitetura do sono em pacientes com transtorno depressivo podem ser divididas em três categorias principais referentes à continuidade do sono, ao sono de ondas lentas e ao sono REM (rapid eye movements). Na primeira, são observados um aumento da latência do sono e de despertares noturnos e o despertar precoce, que resultam na fragmentação do sono e na redução da eficiência deste (Lucchesi et al., 2005). Igualmente, são observados um déficit no sono de ondas lentas e a redução significativa do sono de ondas lentas, notadamente no primeiro período do sono NREM (non-rapid eye movements), o que altera a sua distribuição no decorrer da noite (Stein e Mellman, 2005).

Diversos estudos sugerem que a insônia pode ocorrer nos primeiros estágios do transtorno depressivo, bem como pode antecipá-lo ou ser um sintoma residual da depressão (Chang et al., 1997; Ohayon, 2000; Ford e Cooper-Patrick, 2001). A insônia pode atuar como um importante indicador na avaliação do subsequiente desenvolvimento da depressão recorrente no seguimento de um a três anos (Lucchesi et al., 2005) e tem sido descrita como um fator de risco no surgimento do primeiro episódio depressivo (Riemann e Voderholzer, 2003). prevalent and the mean duration of sleep disorders was higher in relation to depressive disorder.

Key-words: Sleep disorders, sleep initiation and maintenance disorder, disorders of excessive somnolence, depressive disorder.

Assim, a avaliação dos transtornos do sono em pacientes deprimidos é relevante pelos seus valores preventivo, diagnóstico e prognóstico.

O objetivo desta pesquisa foi avaliar as queixas de transtornos do sono em pacientes ambulatoriais com transtorno depressivo de um hospital geral, com a caracterização dos tipos, do tempo de surgimento e da recorrência das queixas de transtornos do sono.

\section{Métodos}

\section{Tipo de estudo e casuística}

O estudo realizado foi do tipo observacional, transversal, com uma amostra não-probabilística de 70 pacientes, 26 homens e 44 mulheres, do Ambulatório de Psiquiatria do Hospital Universitário Onofre Lopes da Universidade Federal do Rio Grande do Norte (UFRN), Natal, Rio Grande do Norte, durante o período de abril a julho de 2005. Essa instituição é considerada um dos principais hospitais públicos de grande porte do Estado do Rio Grande do Norte, para o qual são encaminhados pacientes da Capital e de todo o Estado.

A seleção dos pacientes foi realizada de acordo com os seguintes critérios de inclusão: idade entre 18 e 65 anos e diagnóstico de transtorno depressivo obtido por meio de entrevista clínica com aplicação dos critérios da DSM-IV-R (2000), pelos cinco psiquiatras responsáveis pelo acompanhamento dos pacientes. Neste estudo, todos os pacientes que preencheram os critérios de inclusão foram entrevistados. Os critérios de exclusão foram pacientes com transtorno esquizoafetivo, transtorno bipolar, abuso de substâncias ilícitas e de álcool e transtorno do pânico. Os critérios de exclusão da amostra não abrangeram sintomas da síndrome de apnéia e hipopnéia obstrutiva do sono, de transtornos primários do sono, bem como o uso de betabloqueadores e anti-histamínicos.

\section{Procedimentos}

As entrevistas foram realizadas por um dos pesquisadores deste estudo, devidamente capacitado e treinado na aplicação das metodologias. As escalas de auto-avaliação foram respondidas pelos pacientes com a ajuda de um dos pesquisadores, que explicou de maneira detalhada como responder à Duas escalas utilizadas. 
A pesquisa foi iniciada após a aprovação pelo comitê de ética em pesquisa em seres humanos da UFRN. Todos os indivíduos assinaram o termo de consentimento livre e esclarecido antes de participar da pesquisa e tiveram sua privacidade respeitada.

\section{Instrumentos}

Os pacientes foram entrevistados mediante os seguintes instrumentos de avaliação: ficha de identificação e de avaliação médica, prontuários médicos, questionário de hábitos do sono e Inventário de Depressão de Beck (IDB).

A ficha de identificação e de avaliação médica foi utilizada para acessar os dados sociodemográficos e as informações referentes à presença de comorbidades, à duração do transtorno depressivo e aos episódios depressivos prévios. Os prontuários dos pacientes foram utilizados para conferir os dados de recorrência das queixas e o tempo de surgimento de transtornos do sono, o início de surgimento do transtorno depressivo e a duração em meses do transtorno depressivo e dos transtornos do sono.

O questionário de hábitos do sono consiste em um protocolo padronizado e validado, com 32 itens relacionados aos hábitos de sono, à saúde e ao uso de estimulantes (Andrade, 1992). Esse instrumento foi utilizado para avaliar a presença da insônia e da sonolência excessiva subjetiva. Insônia foi considerada como dificuldade em iniciar o sono, sono fragmentado e/ou despertar precoce pela manhã, ao menos três vezes por semana, por 30 dias (SBS, 2003). Sonolência excessiva subjetiva, por sua vez, foi considerada como sono noturno prolongado e/ou sonolência diurna, ao menos três vezes por semana, por 30 dias.

O IDB é um questionário de auto-avaliação padronizado que permite acessar a gravidade do transtorno depressivo, incluindo atitude e sintomas depressivos (Beck et al., 1961). Esse instrumento apresenta 21 itens, cuja intensidade é indicada por uma pontuação que varia de 0 a 3 . São utilizados os seguintes pontos de corte: < 10: sem depressão ou depressão mínima; de 10 a 18: depressão leve a moderada; de 19 a 29: depressão moderada; de 30 a 63: depressão grave. Neste estudo, foi utilizada a versão validada no Brasil (Gorenstein e Andrade, 1996).

\section{Análise estatística}

Foram realizadas as análises descritivas (frequiências relativa e absoluta, médias e desvios-padrão), o teste $\mathrm{t}$ de Student para amostras pareadas para as comparações entre as médias e a análise de variância (ANOVA), seguida do teste post-hoc de Tukey, para verificar a existência de diferença significativa na pontuação do IDB entre os episódios de transtornos do sono prévios. O nível de significância adotado foi de $5 \%$, e a análise dos dados foi efetivada por meio do software Statistica (versão 6.0).

\section{Resultados}

As características sociodemográficas dos pacientes e a presença de comorbidades são descritas na tabela 1. Um total de $50(71,3 \%)$ pacientes com transtorno depressivo apresentou recorrência das queixas de transtornos do sono. Destes pacientes, todos aqueles que tiveram insônia referiram ter apresentado esse mesmo distúrbio do sono no episódio depressivo prévio. Igualmente, pacientes com sonolência excessiva subjetiva relataram a manifestação desta nos transtornos depressivos anteriores.

Em relação à frequiência das queixas de transtornos do sono, 19 (38\%) apresentaram 1 episódio, 12 (24\%) tiveram 2 episódios, 14 (28\%) relataram 3 episódios e 5 (10\%) referiram mais de 3 episódios de queixas de transtornos do sono relacionados ao transtorno depressivo. A média dos escores de IDB foi de $35,83 \pm 8,85$, e a comparação das médias obtidas nesta escala entre os pacientes com $(38,50 \pm 8,70)$ e sem $(29,60 \pm 7,80)$ recorrência das queixas de transtornos do sono evidenciou diferenças significativas $(t=10,82 ; p<0,05)$. Paralelamente, foram encontradas diferenças significativas $(\mathrm{F}=28,40 ; \mathrm{p}<0,05)$ entre os pacientes que referiram números diferentes de episódios de transtornos do sono.

Tabela 1. Características sociodemográficas e presença de comorbidades dos pacientes.

\begin{tabular}{|c|c|c|}
\hline \multirow{2}{*}{ Variáveis } & \multicolumn{2}{|c|}{ Pacientes $(\mathbf{n}=70)$} \\
\hline & $\mathbf{n}$ & $\%$ \\
\hline Idade & $40,48(12,54)^{*}$ & \\
\hline \multicolumn{3}{|l|}{ Sexo } \\
\hline Homens & 26 & 36,6 \\
\hline Mulheres & 44 & 63,4 \\
\hline \multicolumn{3}{|l|}{ Estado civil } \\
\hline Solteiro & 17 & 24,2 \\
\hline Casado/união consensual & 44 & 62,9 \\
\hline Viúvo & 9 & 12,9 \\
\hline \multicolumn{3}{|l|}{ Nível educacional } \\
\hline Ensino fundamental & 58 & 82,9 \\
\hline Ensino médio & 10 & 14,3 \\
\hline Ensino universitário & 2 & 2,8 \\
\hline \multicolumn{3}{|l|}{ Comorbidades } \\
\hline Hipertensão & 21 & 30 \\
\hline Diabetes & 11 & 15,7 \\
\hline Artrite & 9 & 12,9 \\
\hline Nenhuma & 21 & 30 \\
\hline
\end{tabular}


As médias dos escores de IDB foram mais elevadas quanto maior foi o número de episódios, e os pacientes com mais de três episódios obtiveram a maior média (Figura 1). Os resultados indicaram que 49 (70\%) pacientes apresentaram insônia e 21 (30\%) relataram sonolência excessiva subjetiva. Os dados referentes às características da insônia são descritos na tabela 2 .

Diferenças significativas $(t=6,12 ; \mathrm{p}<0,05)$ foram observadas entre a duração média em meses dos transtornos do sono e do transtorno depressivo. Na amostra estudada, a duração média dos transtornos do sono foi maior que a do transtorno depressivo (Figura 2). Em relação ao uso de medicações, 27 (38\%) pacientes referiram fazer uso de antidepressivos e/ou hipnóticos indutores do sono. Destes pacientes, $9(13,4 \%)$ relataram uso de benzodiazepínicos; 3 (5\%), de amitriptilina; $3(5 \%)$ de nortriptilina, $3(5 \%)$, de sertralina; 2 (3\%), de fluoxetina; e $4(6,6 \%)$ desconheciam qual a medicação utilizada.

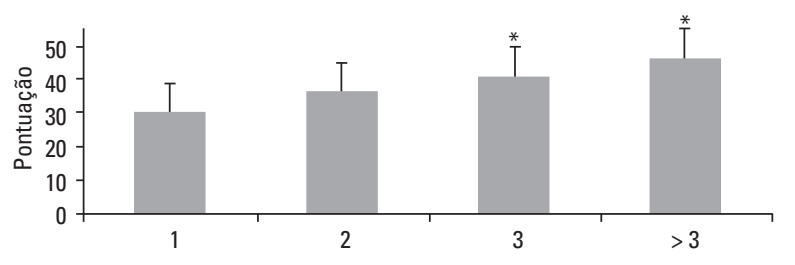

Figura 1. Comparação das médias de pontuações no Inventário de Depressão de Beck entre os pacientes com $1,2,3$ e $>3$ episódios de recorrência de transtornos do sono. ${ }^{*} p<0,05$

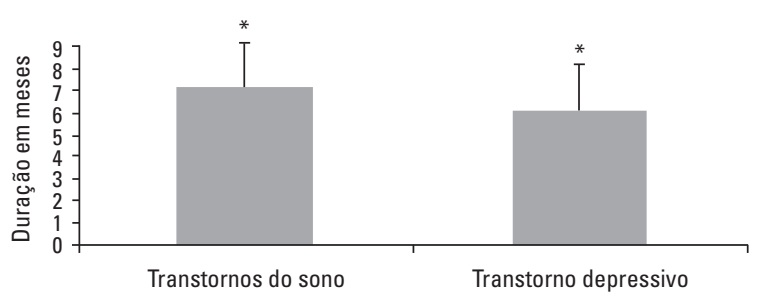

Figura 2. Comparação entre a duração média em meses do transtorno depressivo e dos transtornos do sono nos pacientes. ${ }^{*} p<0,05$

\section{Discussão}

A idade, as características sociodemográficas e a presença de comorbidades observadas nos pacientes indicaram que a maior parcela foi composta por mulheres de meia-idade com menor nível educacional e maior carga de comorbidades crônicas. Esses resultados corroboram positivamente alguns estudos prévios, nos quais foi observada maior prevalência de insônia em adultos de meia-idade e idosos em relação a jovens e referida mais comumente por mulheres no atendi-
Tabela 2. Características das queixas de insônia nos pacientes com transtorno depressivo.

\begin{tabular}{l|c|c}
\hline \multirow{2}{*}{ Variáveis } & \multicolumn{2}{|c}{ Pacientes com insônia (n = 49) } \\
\cline { 2 - 3 } & $\mathbf{n}$ & $\%$ \\
\hline $\begin{array}{l}\text { Dificuldade em iniciar } \\
\text { o sono }\end{array}$ & 48 & 100 \\
\hline $\begin{array}{l}\text { Dificuldade em manter } \\
\text { o sono }\end{array}$ & 46 & 93,8 \\
\hline $\begin{array}{l}\text { Despertar precoce } \\
\text { pela manhã }\end{array}$ & 38 & 77,5 \\
\hline $\begin{array}{l}\text { Redução na duração } \\
\text { total do sono }\end{array}$ & 45 & 91,9 \\
\hline $\begin{array}{l}\text { Duração do sono } \\
\text { Média } \pm \text { DP). }\end{array}$ & $\begin{array}{l}5 \mathrm{~h} 50 \mathrm{~min} \\
(78 \mathrm{~min})^{*}\end{array}$ & \\
\hline
\end{tabular}

mento médico (Voderholzer et al., 2003; Hara et al., 2004). Diversas variáveis sociais e clínicas atuam sobre a qualidade do sono (Voderholzer et al., 2003; Ford e Cooper-Patrick, 2001; Stein e Mellman, 2005). Pacientes com menor nível educacional, presença de comorbidades crônicas (como hipertensão, diabetes e artrite reumatóide) e menor apoio social apresentam comparativamente pior perfil de sono em relação a indivíduos sadios e com um melhor nível socioeconômico (CanoLozano et al., 2003). Assim, esses fatores podem apresentar um papel patogênico sobre o padrão de sono dos pacientes, o que torna necessária a investigação criteriosa daqueles durante a avaliação clínica das queixas de transtornos do sono.

Nesta pesquisa, foi observada elevada recorrência das queixas de transtornos do sono, e os pacientes com maior número de episódios apresentaram maior pontuação obtida na IDB, o que sugere episódio depressivo grave. Uma explicação para esses resultados é que as queixas de transtornos do sono constituíram parte dos critérios diagnósticos de transtornos do humor (DSM-IV-R, 2000). De maneira simultânea, tais queixas podem representar um fator de vulnerabilidade para o desenvolvimento do transtorno depressivo, que se torna de forma paulatina mais grave a cada novo quadro de depressão. Isso se deve principalmente às alterações mais pronunciadas do sono REM encontradas em pacientes com depressão recorrente (Thase et al., 1995). As alterações do sono podem, portanto, atuar como um marcador psicobiológico no desenvolvimento de um novo episódio depressivo (Riemann et al., 2001).

A diferença entre a duração média em meses dos transtornos do sono e do transtorno depressivo pode sugerir uma antecipação das alterações do sono em relação ao episódio depressivo. Todavia, esse resultado não é conclusivo, pois os transtornos do sono podem permanecer mais tempo que os outros sintomas de- 
pressivos, podendo ser um resíduo depressivo (Stein e Mellman, 2005).

A maior implicação clínica desses dados é que o monitoramento adequado das queixas de sono pode permitir uma intervenção terapêutica em um período anterior ao desenvolvimento do transtorno depressivo, o que realça seu valor preventivo (AASMR, 1999; Morin, 2005).

Houve predominância das queixas relacionadas à insônia, como dificuldades em iniciar o sono; menor duração do sono, com fragmentação deste; e despertar precoce pela manhã. A insônia é, em muitos casos, concomitante a algumas condições médicas, como transtornos psiquiátricos - principalmente em episódios depressivos - transtornos cardiopulmonares e condições associadas a queixas somáticas crônicas, como artrite reumatóide. Atualmente, esse tipo de insônia é denominado de comórbida, o que permite uma conclusão mais adequada sobre a natureza dessas associações e a direção de causalidade que existe entre estas (NIH-NIMH, 2005).

Em um estudo prévio, foi realizada a avaliação dos padrões de sono, na qual se observou que 15 dos 36 sintomas depressivos avaliados e 9 das 19 variáveis eletroencefalográficas do sono analisadas explicam em até $95 \%$ a relação entre a insônia e a depressão (Thase et al., 1995). Paralelamente, o uso de técnicas de neuroimagem tem demonstrado que os transtornos do sono concomitantes ao transtorno depressivo podem decorrer de alterações em áreas cerebrais específicas (Nofzinger, 2005). Desta forma, a relação intrínseca entre os mecanismos de regulação do sono e do humor é responsável pelas alterações do padrão de sono presentes no transtorno depressivo (Cano-Lozano et al., 2003; Benca, 2005).

Neste estudo, foi observada uma prevalência de $30 \%$ de sonolência excessiva subjetiva, algo comparativamente superior ao encontrado em pacientes com transtorno depressivo, cuja prevalência é geralmente em torno de 10\% (Bittencourt et al., 2005). Em um estudo prévio, foi observado que pacientes com duração total do sono de 8 horas ou mais apresentaram mortalidade significativamente elevada em comparação a pacientes que possuíram menor duração total do sono (Kripke et al., 2002). Esse dado realça a importância da adequada avaliação das queixas de sonolência excessiva subjetiva no atendimento clínico. A presença de apnéia e/ou hipopnéia obstrutiva do sono e de transtornos primá- rios do sono, bem como o uso de betabloqueadores e anti-histamínicos, pode colaborar com sintomas de sonolência excessiva subjetiva na amostra estudada. Assim, as causas da sonolência nesses pacientes são incertas. Em estudos futuros, é sugerida a pesquisa desses fatores na anamnese dos hábitos do sono de pacientes com transtornos depressivos e queixas de transtornos do sono.

Em relação às limitações do estudo, deve ser salientado o seu desenho transversal, que não permite um adequado estabelecimento da relação causa-efeito dos achados. Outro fato foi o uso de antidepressivos e/ou benzodiazepínicos por parte da amostra. Isso em parte limita a extensão dos resultados obtidos, pois o uso dessas medicações pode acarretar um leque de efeitos sobre o sono, que inclui desde pior percepção do sono até sonolência excessiva subjetiva (Moreno et al., 1999; Walsh et al., 2005). A presença de apnéia e/ou hipopnéia obstrutiva do sono consiste em um viés de informação, pois está bem estabelecido que estas são com frequiência encontradas na depressão, principalmente entre mulheres de meiaidade. (Bittencourt et al., 2005). Outrossim, a realização de polissonografia nos pacientes depressivos com queixas de transtornos do sono deve ser ressaltada, na medida em que permite um registro mais fidedigno dos transtornos do sono. Futuros estudos com desenho longitudinal com a utilização de um grupo-controle (estudo de casocontrole) e a inclusão de pacientes livres de medicação antidepressiva e/ou benzodiazepínica são sugeridos para melhor avaliar a recorrência e o tempo de surgimento dos transtornos do sono em pacientes deprimidos.

Como conclusão, neste estudo, a recorrência das queixas de transtornos do sono nos pacientes foi elevada e significativamente associada a pontuações mais altas no IDB. Em paralelo, foi observado que a duração média dos transtornos do sono foi maior em relação àquela do transtorno depressivo. Houve prevalência da insônia, e a sonolência excessiva subjetiva foi maior em relação a estudos prévios.

\section{Agradecimentos}

Os autores agradecem a colaboração proporcionada pelo Departamento de Psiquiatria do Hospital Universitário Onofre Lopes da UFRN.

\section{Referências bibliográficas}

American Academy of Sleep Medicine - International Classification of Sleep Disorders, Second Edition:Diagnostic and Coding Manual. Westchester, IL: American Academy of Sleep Medicine, 2005.

American Academy of Sleep Medicine Review - Nonpharmacological treatment of chronic insomnia. Sleep 22: 1134-1156, 1999.

American Psychiatric Association - Diagnostic and Statistical Manual of Mental Disorders - TR, 4.ed. American Psychiatric Association, Washington, DC, 2000.
Andrade, M.M.; Benedito-Silva, A.A.; Menna-Barreto, L. - Correlations between morningness-eveningness character, sleep habits and temperature rhythm. Braz J Med Biol Res 25 (8): 835-839, 1992.

Beck, A.T.; Ward, C.H.; Mendelson, M.; Mock, J.; Erbaugh, J. - An inventory for measuring depression. Arch Gen Psychiatry 4: 561-571, 1961.

Benca, R.M. - Mood disorders. In: Kryger, M.H.; Roth, T.; Dement, W.C. (Eds.) Principles and Pratice of Sleep Medicine. 4.ed., WB Saunders, Philadelphia, pp. 1311-1326, 2005. 
Bernert, R.A.; Joiner, T.E.; CukRowicz, K.C. - Suicidality and sleep disturbances. Sleep 28 (9): 1135-1141, 2005.

Bittencourt, L.R.A.; Silva, R.S.; Santos, R.F.; Pires, M.L.N.; Mello, M.T. - Excessive daytime sleepiness. Rev Bras Psiquiatr 27 (1): 16-21, 2005.

Bonnet, M.H.; Arand, D.L. - Hyperarousal and insomnia. Sleep Med Rev 1: 97-108, 1997.

Cano-Lozano, M.C.; Espinosa-Fernandez, L.; Miro, E.; Buela-Casal, G. - Una revisión de las alteraciones del sueño en la depressión. Rev Neurol 36 (4): 3666-3375, 2003.

Chang, P.P.; Ford, D.E.; Mead, L.A. - Insomnia in young men and subsequent depression. The Johns Hopkins precursors study. Am J Epidemio/ 146: 105-114, 1997.

Ford, D.E.; Cooper-Patrick, L. - Sleep disturbances and mood disorders: an epidemiologic perspective. Depress Anxiety 14: 3-6, 2001.

GorensteIn, C.; AndRADE, L. - Validation of a portuguese version of the Beck Depression Inventory and the State-Trait anxiety inventory in Brazilian subjects. Braz J Med Biol Res 29: 453-457, 1996.

Hara, C.; Lopes Rocha, F.; Lima-Costa, M.F. - Prevalence of excessive daytime sleepiness and associated factors in a Brazilian community: the Bambui study. Sleep Med 5: 31-36, 2004.

Kripke, D.; Garfinkel, L.; Wingard, D.L.; Kaluber, M.R.; MarleR, M.R. - Mortality associated with sleep duration and insomnia. Arch Gen Psychiatry 59: 131-136, 2002.

LucchesI, L.M.; Pradella-Hallinan, M.; Lucchesi, M.; Moraes, W.A.S. - 0 sono em transtornos psiquiátricos. Rev Bras Psiquiatr 27 (1): 27-32, 2005.

Moreno, R.A.; Moreno, D.H.; Soares, M.B.M. - Psicofarmacologia dos antidepressivos. Rev Bras Psiquiatr 21: 24-40, 1999.

Morin, C. - Psychological and behavioral treatments for primary insomnia. In: Kryger, M.H.; Roth, T.; Dement, W.C. (Eds.) Principles and Practice of Sleep Medicine. 4.ed., WB Saunders, Philadelphia, pp. 726-737, 2005.
National Institutes of Health. State of the Science State Conference. Sleep 28: 1049-1057, 2005.

Nofzinger, E.A. - Neuroimaging and sleep medicine. Sleep Med Rev 9: 157-172, 2005.

Ohayon, M.M. - Epidemiology of insomnia: what we know and what we still need to learn. Sleep Med Rev6 (2): 97-111, 2000.

Riemann, D.; Berger, M.; Voderholzer, U. - Sleep and depression - results from psychobiological studies: an overview. Biol Psychiatry 57: 67-103, 2001.

Riemann, D.; Voderholzer, U. - Primary insomnia: a risk factor to develop depression? J Affect Disord 76 (1-3): 255-259, 2003.

Sociedade Brasileira de Sono. I Consenso Brasileiro sobre Insônia. Hypnos 4: 3-45, 2003.

Stein, M.B., Mellman, T.A. - Anxiety Disorders. In: Kryger, M.H.; Roth, T.; Dement W.C. (Eds.) Principles and Practice of Sleep Medicine. 4.ed., WB Saunders, Philadelphia, pp. 1297-1311, 2005.

Thase, M.E.; Fasicka, A.L.; Berman, S.R.; Simon, A.D.; Reynolds, C.F. - Electroencephalographic sleep profiles in single-episode and recurrent unipolar forms of major depression: I. Comparison during acute depressive states. Biol Psychiatry 38: 99-108, 1995.

Vaughn, B.V.; D'Cruz, O.N.F. - Cardinal Manifestations of Sleep Disorders. In: Kryger, M.H.; Roth, T.; Dement W.C. (Eds.) Principles and Practice of Sleep Medicine. 4.ed., WB Saunders, Philadelphia, pp. 594-601, 2005.

Voderholzer, U.; Al-Shajlawi, A.; Weske, G.; Felge, B.; Riemann, D. - Are there gender differences in objective and subjective sleep measures? A study of insomniacs and healthy controls. Depress Anxiety 17: 162-72, 2003.

Walsh, J.K.; Roehrs, T.; Roth, T. - Pharmacologic Treatment of Primary Insomnia. In: Kryger, M.H.; Roth, T.; Dement, W.C. (Eds) Principles and Practice of Sleep Medicine. 4.ed., WB Saunders, Philadelphia, pp. 749-760, 2005. 